\title{
Quantification of Solid Residues by Raman Spectroscopy
}

\author{
Esau Araiza-Reyna ${ }^{1}$, Roberto Sato-Berrú ${ }^{2}$, América Vázquez-Olmos ${ }^{2}$ \\ ${ }^{1}$ Facultad de Ciencias, Universidad Autónoma de San Luis Potosí, \\ Av. Salvador Nava Martínez s/n, Zona Universitaria, San Luis Potosí, México \\ ${ }^{2}$ Centro de Ciencias Aplicadas y Desarrollo Tecnológico, Universidad Nacional Autónoma de México, \\ Circuito Exterior s/n, Ciudad Universitaria, Delegación Coyoacán, México D.F., México \\ Email: roberto.sato@ccadet.unam.mx
}

Received May 17, 2013; revised June 22, 2013; accepted July 19, 2013

Copyright (C) 2013 Esau Araiza-Reyna et al. This is an open access article distributed under the Creative Commons Attribution License, which permits unrestricted use, distribution, and reproduction in any medium, provided the original work is properly cited.

\begin{abstract}
We suggest a mathematical route which has the capability to obtain quantitative information of solid residues on a substrate of aluminum, in which the evaporation of the deposited micro-drops has the form of coffee rings. In this job, the glycine aminoacid was used as probe molecule. We suppose that the number of moles present on the study sample is proportional to the Raman signal and to the experimental parameters of the used Raman system. Then, the mass and the number of molecules can be determined with these data. We showed that the mathematical expression is simple and elegant for its implementation. On the other hand, we have applied and demonstrated that the technique of principal component analysis (PCA) can be used to obtain quantitative information of the sample. Our results show quantitative analysis of glycine residues between $10^{-11}$ to $10^{-15} \mathrm{~g}$.
\end{abstract}

Keywords: Quantitative Raman; Aminoacids; Glycine; Solid residues; Drop Coating Deposition Raman; Principal Component Analysis

\section{Introduction}

Glycine is the smallest molecule between the aminoacids; it has the simplest chemical structure $\left(\mathrm{NH}_{2} \mathrm{CH}_{2} \mathrm{COOH}\right)$ and it is part of various proteins in living of things [1-3]. Its function inside the organism is as inhibiting neurotransmitter in the central nervous system that acts as door of chlorine channel [4]. Because of the clinical significance of the proteins and aminoacids in the body, it is important to develop new and better techniques for your detection and quantification. Raman and infrared spectroscopy offers potentially powerful and noninvasive technique for analysis of biomolecules [5-7].

Raman spectroscopy has made great progress and partly because of its versatility of analysis. For example, the samples require no preparation in comparison with other techniques. However, there is a lack of information of this technique in the quantitative analysis, especially in the quantification of solid residues. Raman spectroscopy (macroRaman) is a technique that requires relatively large quantities of samples which has a detection limit of the order of concentrations greater than $10^{-3} \mathrm{M}$. Raman microscopy (microRaman) can detect small quantities of sample but still a high concentration $\left(10^{-5}\right.$ $\mathrm{M})$ is necessary. This technique uses a combination of
Raman technique and a conventional optical microscope to allow a micrometric characterization of the material [6,7]. The Drop Coating Deposition Raman (DCDR) method, based in the aliquots deposition on hydrophobic substrates, allows obtaining solid residues in the form of coffee rings. This technique has a detection limit about of $10^{-6} \mathrm{M}[8-10]$. For the detection of substances diluted to more low concentrations, methods such as the SERS (Surface Enhanced Raman Scattering) have been developed $[11,12]$, which involve complex metallic substrates or synthesis of colloidal suspensions $[13,14]$. The SERS phenomenon is the enhancement of Raman signal from molecules in the presence of metallic nanostructures which has a detection limit around of $10^{-10} \mathrm{M}[15,16]$.

All the quantification jobs that we have consulted showed a linear trend between the Raman signal and the concentration of the sample. This trend makes that this technique can be predictable in ideal conditions. Therefore, we are convinced that this work complements and helps to obtain quantitative information of various solid residues. Here, we have used the micro-drop deposition method determining the amount of material that is transferred to a substrate and we propose a relationship math in which the Raman signal is proportional to the number of molecules presents in the area of analysis. Moreover, 
we have introduced and demonstrated that technique of principal component analysis can be used to obtain quantitative information and discrimination of data in form simultaneous.

\section{Materials and Methods}

\subsection{Raman Spectroscopy}

We recorded Raman spectra with an Almega XR dispersive Raman spectrometer. An Olympus microscope (50X and 0.85 NA: numerical aperture) was used both for focusing the laser on solid samples (residues) and for collecting the scattered light in a 180-degree backscattering configuration. In addition, Raman spectra were accumulated over $25 \mathrm{~s}$ with a resolution of $\sim 4 \mathrm{~cm}^{-1}$, the excitation source was $532 \mathrm{~nm}$ radiation from a Nd: $\mathrm{YVO}_{4}$ laser (frequency-doubled) and the laser power on the sample was $10 \mathrm{~mW}$.

On aluminum substrates, aliquots of $1 \mu \mathrm{L}$ were deposited and dried at $40^{\circ} \mathrm{C}$. These drops produced residues with form of coffee rings. Eight spectra were measured around of each ring. In first approximation, we have considered a homogeneous area $\left(A_{H}\right)$ of $7.8 \times 10^{-7} \mathrm{~m}^{2}$ and the laser spot area $\left(a_{s}\right)$ of $5.2 \times 10^{-13} \mathrm{~m}^{2}$. We have found the geometric ratio $A=\left(a_{s} / A_{H}\right)$ that was of 6.6 $\times 10^{-7}$.

We seek to test the feasibility of implementing the microRaman technique for quantitative analysis using conventional substrates such as glass; silicon and aluminum foil [17]. The glycine aminoacid (99\%) was purchased of Fluka and prepared in different concentrations from $0.133 \mathrm{M}(10000 \mathrm{ppm})$ to $1.33 \times 10^{-5} \mathrm{M}(1 \mathrm{ppm})$ in tri-distilled water (QuimiNet, Mexico). All samples used in this work were used as received.

\subsection{Theoretical Analysis}

We have studied the following relationship:

$C_{i} V_{i} \rightarrow A_{H} \rightarrow a_{s}$, where we considered that $V_{i}$ and $C_{i}$ are the volume and the concentration initial (sample of reference); $A_{H}$ is the area in a homogeneous configuration and $a_{s}$ is the area of the laser spot used in the Raman experiment. For an objective (microRaman configuration), we have used the following relation: $d=1.22 / N A$ as the diameter of a circle. Where $\lambda$ is the wavelength of laser and $N A$ is the numerical aperture of the objective, see Figure 1. Whereas a Gaussian beams, we determined the area of the laser spot [18].

However, we have noted that the number of moles " $n$ " does not remain constant $\left(n_{\text {initial }}>n_{\text {Homogeneous }}>n_{\text {spot }}\right)$, this changes as the volume of study decreases. Based on these considerations, a mathematical expression can be obtained for number of moles that exist within the laser spot (area or volume):

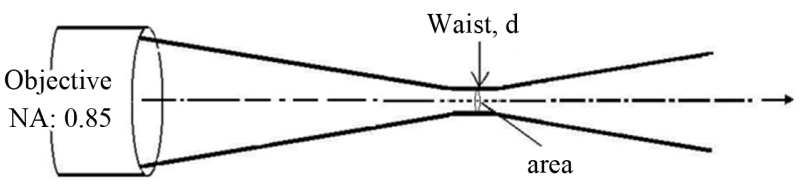

Figure 1. Gaussian profile of laser beam. " $d$ " is the beam waist.

$$
n_{s}=A * C * V
$$

where $A=G_{s} / G_{H}$ is the ratio between the geometry of the laser spot and the homogeneous geometry considered in the experiment, $G_{s}$ can represent the volume or the area of the laser spot, and $G_{H}$ can represent the volume or the residual area in a homogeneous distribution of the study sample. $C$ is the concentration of the sample in Molarity and $V$ is the volume of study in liters. If the number of moles in a system is known, the sample mass and the number of molecules can be determined with the following relations:

$$
\begin{gathered}
g=n_{s} * W \\
N_{\text {molecules }}=n_{s} * N_{\text {Avog }}
\end{gathered}
$$

where, $W$ is the molecular weight of the study sample and $N_{A v o g}$ is the Avogadro's number $\left(6.022 \times 10^{23}\right.$ $\left.\mathrm{mol}^{-1}\right)$.

On the other hand, theoretically, it is known that the Raman intensity is mainly proportional to the intensity of the excitation source and to the number of molecules in the sample volume being probed with the Raman instrument $[19,20]$. Experimentally, it is known that the intensity of a Raman spectrum is directly proportional to the concentration. In this paper, considering the previous premises, we will propose and define that the Raman signal is proportional to the number of molecules by using the following simple relationship:

$$
I_{R}=M * i
$$

where $M$ represents the number of elementary entities and $i$ represents the intrinsic Raman emission, which is individual and representative of the material of study under the experimental conditions. If we assume that $M=n_{s}$, where $n_{s}$ represents the number of moles present within the laser spot (area). Using Expression (1), the unitary intensity can be expressed as $i=I_{R} / n_{s}$. Similarly, it can be assumed that it also satisfies the relation $I_{r}=n * i$, where $I_{r}$ represents an arbitrary Raman signal. Therefore, the number of moles " $n$ " can be determined in an arbitrary Raman signal with the following relation:

$$
n=I * n_{s}
$$

where, $I=I_{r} / I_{R}$ is the ratio between the arbitrary and reference Raman intensity. We observed that $I$ varies between $0 \leq I \leq 1$. Expression (5) represents the mathe- 
matical general approach proposed in this paper.

For solid residues, we must consider the following expression:

$$
n=I * A * C * V
$$

where $A=a_{S} / A_{H}, C=C_{i}, V=V_{\mathrm{nL}}$ or $V_{\mu \mathrm{L}}$; where $a_{S}$ and $A_{H}$ are the laser spot and the homogeneous distribution areas, respectively, of the study sample; $C_{i}$ is the initial (reference) concentration; and $V_{\mathrm{nL}}$ or $V_{\mu \mathrm{L}}$ is the aliquot in nanoliters or microliters deposited on the substrate.

However, it is observed that solid residues acquire habitually a heterogeneous distribution. Therefore, the $n$ (experimental) value and the expected value $\left(n_{s}\right)$ can be related by

$$
n \approx n_{\text {heterogeneous }}=h * n_{s}
$$

where $h \geq 1$ and it is a scaling factor on a more real measure of the studied sample. This represents the different distributions of the sample when this evaporates on the analysis substrate. For example: $h=1$, the distribution is $100 \%$ coincidental with the homogeneous distribution; $h=2$, the distribution is $50 \%$ coincidental; $h=$ 5 , the distribution is $20 \%$ coincidental, and so on. We implemented all these expressions in an excel worksheet.

\section{Results and Discussion}

We have used the Equations (1)-(3) for analysis of glycine residues and have obtained the expected values in a micro-Raman experiment as the moles number, the mass amount and the molecules number present in the laser spot, see Table 1. Here, we have considered that the volume of $1 \mu \mathrm{L}$ produced homogeneous residual areas (c.a. $1 \mathrm{~mm}$ ) which obviously, they are larger than the area of laser spot. However, we have observed that the residue acquired a heterogeneous distribution which implies a lesser probability or certainty of that the residue or sample in study is found within the laser spot. Therefore, we note that in the microRaman configuration, the detection limit by glycine was found in a concentration of $10^{-5} \mathrm{M}$. However, we have found $10^{-16} \mathrm{~g}$ or $10^{6}$ mole-

Table 1. Expected values in the homogeneous case (using the Equations (1)-(3)): initial concentration (molaridad); number of moles; mass amount and number of molecules.

\begin{tabular}{cccc}
\hline Molarity & Moles & Grams & Molecules \\
\hline $1.3 \times 10^{-1}$ & $1.08 \times 10^{-13}$ & $8.13 \times 10^{-12}$ & $6.50 \times 10^{10}$ \\
$1.3 \times 10^{-2}$ & $1.08 \times 10^{-14}$ & $8.13 \times 10^{-13}$ & $6.50 \times 10^{9}$ \\
$1.3 \times 10^{-3}$ & $1.08 \times 10^{-15}$ & $8.13 \times 10^{-14}$ & $6.50 \times 10^{8}$ \\
$1.3 \times 10^{-4}$ & $1.08 \times 10^{-16}$ & $8.13 \times 10^{-15}$ & $6.50 \times 10^{7}$ \\
$1.3 \times 10^{-5}$ & $1.08 \times 10^{-17}$ & $8.13 \times 10^{-16}$ & $6.50 \times 10^{6}$ \\
\hline
\end{tabular}

cules into the laser spot. Finally, these equations us predicts that in $10^{-12} \mathrm{M}$, we should be detecting a single molecule. This range of concentration can be observed in work different, especially on SERS and SMD (Single Molecular Detection) jobs [21,22].

Due to heterogeneity in the drying of the sample, we have observed light variations in the intensity of Raman signal in several areas of the same solid residue. The most common solution to this problem is to use a standard sample, wherein a homogeneous distribution and that the concentration of the sample is assumed to be proportional to the ratio of the sample peak height to the standard peak height. The instrumental variation affect the signal measured from the standard sample in the same way as the problem sample signal, this method can achieve excellent quantitative results as will be seen in this work.

For this analysis, we have obtained eight Raman spectra from different areas of the residue and at the different concentrations. We have chosen the peak at $1328 \mathrm{~cm}^{-1}$ for the analysis that is a typical vibration of glycine aminoacid. Figure 2 shows the number of moles (geometric figures) and mass amount (black points) which they can be obtained in a microRaman experiment. For this exercise, we must know the reference Raman intensity $\left(I_{R}\right)$ and the intensity of the problem sample $\left(I_{r}\right)$, then we have used the Equation (6) for determine the number of moles. The concentration $10^{-1} \mathrm{M}$ is a good point of reference for this range of concentrations.

Figure 3 shows the deviation values, $D=(($ experiment - expected $) /$ expected $) * 100$, between the expected and experimental data. Because of experimental complexity, we have observed that a homogeneous distribution is not adequate. Therefore, we have had that considerer a heterogeneous distribution by the analysis of solid residues. The Expression (7) is implemented and used when the value of " $h$ " exceeds a value

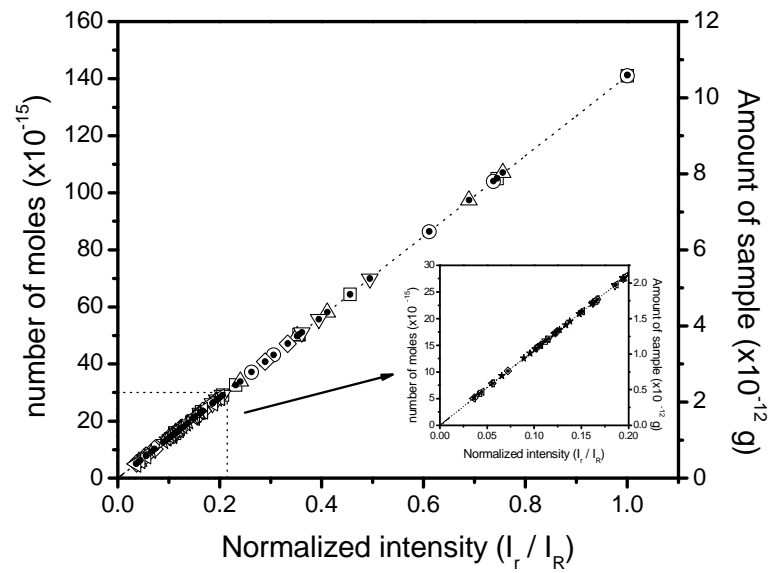

Figure 2. Expected (line) and experimental (geometric figures) data for glycine sample. 

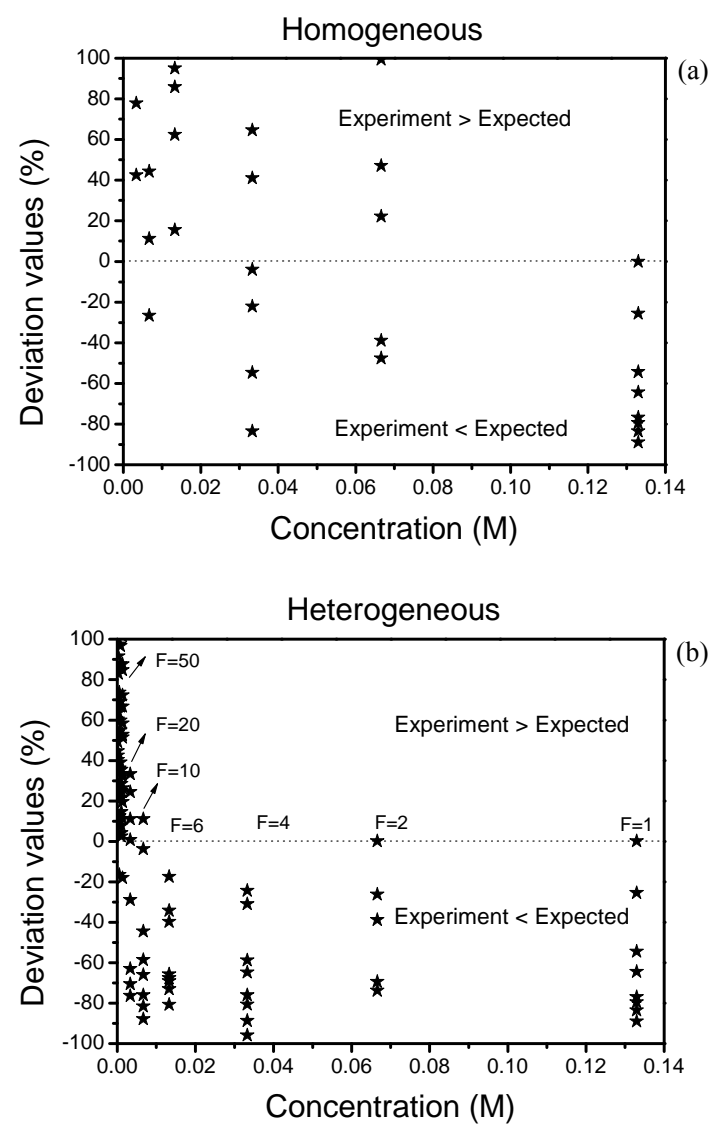

Figure 3. Deviation values of data, (a) homogeneous distribution and (b) heterogeneous distribution.

of 10 . The deviation rises above of $100 \%$ and in this case, we must take a new reference. The results are shown in Figures 3(a) and (b).

The quantitative analysis of solid residues on substrates is not easy task. Here, we show that it is feasible obtain quantitative information changing the initial concentration (reference) to another point and detect other concentration using solid residues as $10^{-3} \mathrm{M}$. Figure 4 shows the number of moles that they can be obtained in a microRaman experiment. For this exercise, we must know the reference Raman intensity $\left(I_{R}\right)$ and the intensity of the problem sample $\left(I_{r}\right)$, then we use the equation 6 for determine the number of moles. The concentration $10^{-3} \mathrm{M}$ is a good point of reference for this new range of concentrations. These results give us a first approximation the quantitative development of values in more reliable.

Figure 5 shows the deviation values between the expected and experimental data. Equally, because of experimental complexity, we have observed that a homogeneous distribution is not adequate in this experiment. Therefore; we have had that considerer a heterogeneous distribution by the analysis of solid residues. Again, the Expression (7) is used when the value of " $h$ " exceeds a

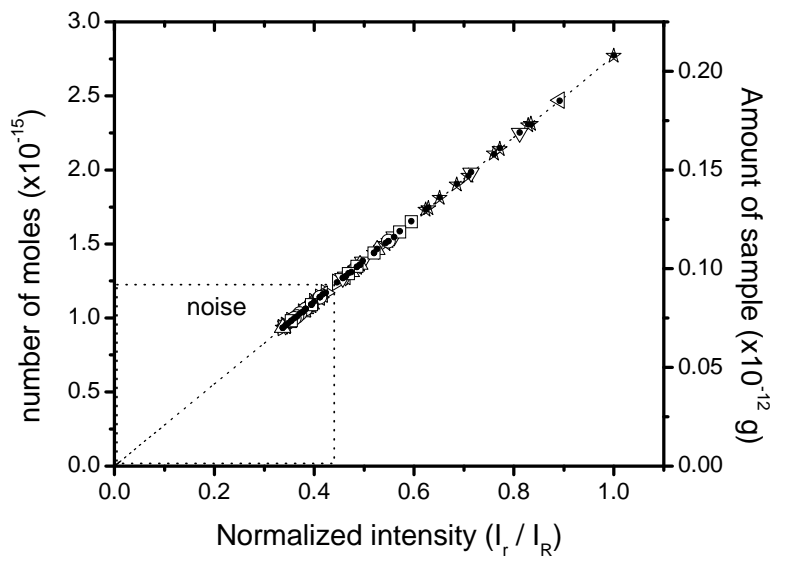

Figure 4. Expected (line) and experimental (geometric figures) data for glycine sample.
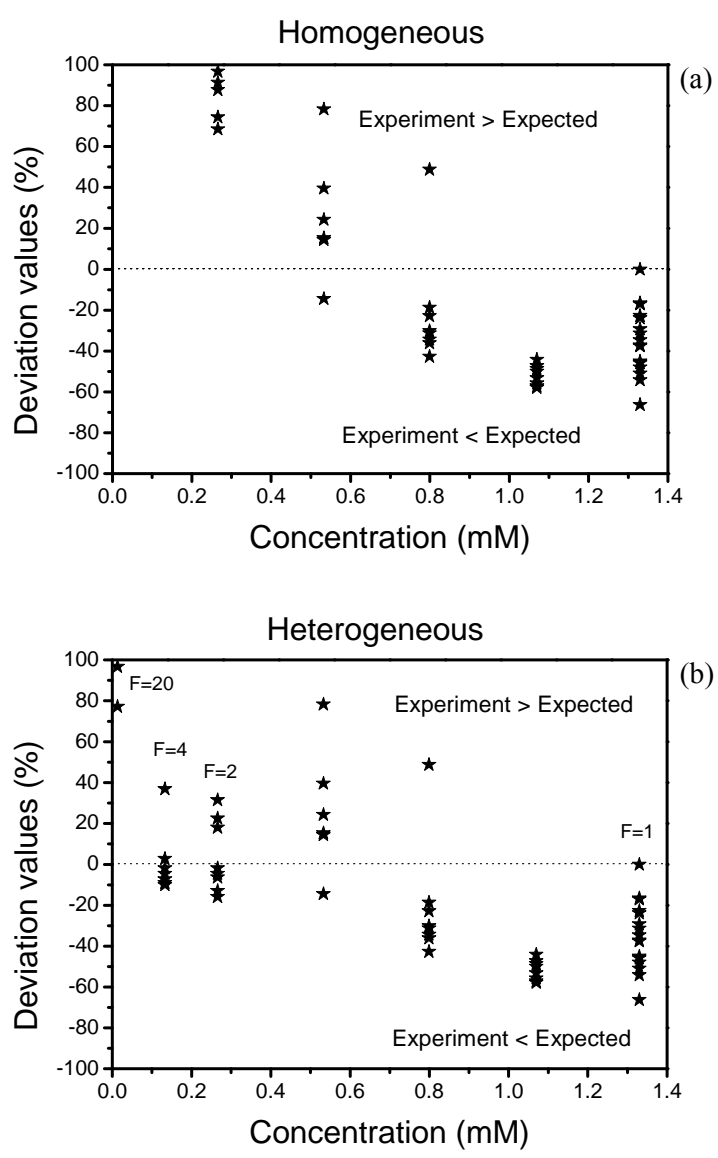

Figure 5. Deviation values of data, (a) homogeneous distribution and (b) heterogeneous distribution.

value of 10 . The deviation rises above $100 \%$ and in this case, we must take a new reference. The results are shown in Figures 5(a) and (b).

The previous graphs were only using a single Raman peak. We supplement our analysis with the PCA multivariate analysis method (see Figure 6). Here, each point 


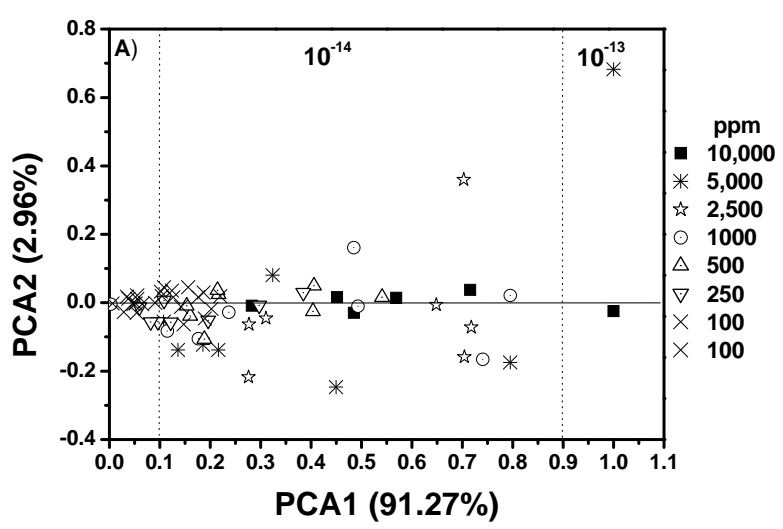

(a)

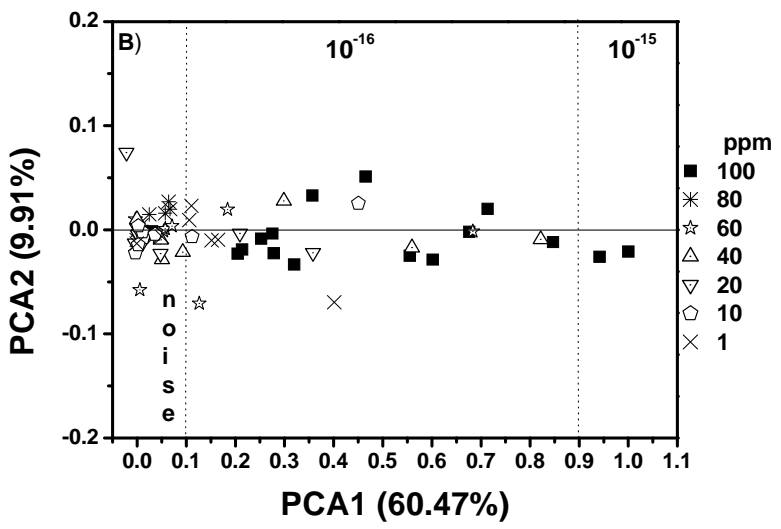

(b)

Figure 6. Scores of (a) PCA1 (91.27\%) vs PCA2 (2.96\%) between $10^{-13}$ and $10^{-14}$ moles. (b) PCA1 (60.47\%) vs PCA2 $(9.91 \%)$ between $10^{-15}$ and $10^{-16}$ moles.

represents a Raman spectrum and also, the initial concentration is indicated. Each drop generated a residual distribution heterogeneous, and therefore, different intensities of the Raman signals were obtained. Our proposal is that each Raman signal is proportional to the mass amount of the sample in study. In addition, we have modified the $\mathrm{x}$ axis (PCA 1) so that it matches the ratio of intensities. With this technique we can determine ranges of measurable values and discern spectral differences from the measurements. We could identify interactions between the sample and substrate; we could discern between Raman and SERS spectrum in the same molecule and we could determine the orientation of the microcrystal formed. The PCA is an excellent tool to differentiate molecules in a mixture.

The experimental data show that our quantification proposal is viable and very easy to implement. Under our experimental conditions, we can assign the amount of sample that could be obtained in a homogeneous distribution and the studied analyte concentration could be obtained through the Raman signal with enough precision. With respect to the problem of heterogeneity of some experimental arrangements, we observed that they can be minimized with the acquisition of a good point of reference or adequate initial concentration. The purpose of considering a tunable initial concentration is based on the idea that it is not right to measure objects with the same tool when they have large differences in size. Nevertheless, the development of standard samples was a problem in this work; therefore, we are working on it. We are aware that there is appropriate technology to develop sampling standards that will help us in the development of more reliable quantitative measures through the Raman technique since this technique is used in a wide variety of applications, and also because it presents various benefits.

This is the first work that presents a math relationship that relates directly the Raman signal to the amount of studied sample. Also, we think that it could be used to obtain quantitative information in the SERS technique.

\section{Conclusion}

In this work, we have demonstrated and applied a simple mathematical route for obtaining quantitative information from Raman signals. This approach, along with the PCA technique, helped us to assign quantitative values to the Raman intensity. We showed that it is feasible to consider a homogeneous distribution for quantitative analysis. However, we have observed that we can also obtain quantitative information in a heterogeneous distribution. The aluminum substrate, for the solid residue analysis, has showed be a promising tool, as well as in the study of aminoacids. The samples deposited on the aluminum substrate have generated residues of coffee rings. In summary, we showed a general equation that could help us to quantify any type of specimen using the Raman technique.

\section{Acknowledgements}

The authors acknowledge the PAPIIT IA100813 (UNAM) project. The ICyTDF and Cofor for their support, but special acknowledge from E. A. Araiza-Reyna at CONACYT by their support with registration number 168944.

\section{REFERENCES}

[1] H. Betz and B. Laube, "Glycine Receptors: Recent Insights into Their Structural Organization and Functional Diversity," Journal of Neurochemistry, Vol. 97, No. 6, 2006, pp. 1600-1610. doi:10.1111/j.1471-4159.2006.03908.x

[2] B. Matilla, J. L. Mauriz, J. M. Culebras, J. GonzálesGallegos and P. González, "Glycine: A Cell-Protecting Anti-Oxidant Nutrient," Nutrición Hospitalaria, Vol. 17, No. 1, 2002, pp. 2-9. 
[3] J. Loertscher and V. Minderhout, "Foundations of Biochemistry," Pacific Crest, Plainfield, 2011.

[4] S. Yamashina, K. Ikejima, I. Rusyn and N. Sato, "Glycine as a Potent Anti-Angiogenic Nutrient for Tumor Growth," Journal of Gastroenterology and Hepatology, Vol. 22, No. S1, 2007, pp. S62-S64.

[5] D. A. Skoog, F. J. Holler and S. R. Crouch, "Principios de Análisis Instrumental," Cengage Learning Editores, Mexico City, 2008.

[6] J. J. Laserna, "Modern Techniques in Raman Spectroscopy," John Wiley and Sons Ltd., Chichester, 1996.

[7] Y. Shi and L. Wang, "Collective Vibrational Spectra of $\alpha$ and $\gamma$-Glycine Studied by Terahertz and Raman Spectroscopy," Journal of Physics D: Applied Physics, Vol. 38, No. 19, 2005, pp. 3741-3745. doi: $10.1088 / 0022-3727 / 38 / 19 / 024$

[8] D. Zhang, Y. Xie, M. F. Mrozek, C. Ortiz, V. J. Davisson and D. Ben-Amotz, "Raman Detection of Proteomic Analytes," Analytical Chemistry, Vol. 75, No. 21, 2003, pp. 5703-5709. doi:10.1021/ac0345087

[9] Y. Xie, Y. Jiang and D. Ben-Amotz, "Detection of Amino Acid and Peptide Phosphate Protonation Using Raman Spectroscopy," Analytical Biochemistry, Vol. 343, No. 2, 2005, pp. 223-230. doi:10.1016/j.ab.2005.05.038

[10] C. Ortiz, D. Zhang, Y. Xie, A. E. Ribbe and D. BenAmotz, "Validation of the Drop Coating Deposition Raman Method for Protein Analysis," Analytical Biochemistry, Vol. 353, No. 2, 2006, pp. 157-166. doi:10.1016/j.ab.2006.03.025

[11] F. J. Garcia-Vidal and J. B. Pendry, "Collective Theory for Surface Enhanced Raman Scattering," Physycal Revies Letters, Vol. 77, No. 6, 1996, pp. 1163-1166. doi:10.1103/PhysRevLett.77.1163

[12] J. S. Suh and M. Moskovits, "Surface-Enhanced Raman Spectroscopy of Amino Acids and Nucleotide Bases Adsorbed on Silver," Journal of the American Chemical Society, Vol. 108, No. 16, 1986, pp. 4711-4718. doi:10.1021/ja00276a005

[13] R. Sato-Berrú, R. Redón, A. Vázquez-Olmos and J. M. Saniger, "Silver Nanoparticles Synthesized by direct Photoreduction of Metal Salts. Application in SurfaceEnhanced Raman Spectroscopy," Journal of Raman Spectroscopy, Vol. 40, No. 4, 2009, pp. 376-380. doi:10.1002/jrs. 2135

[14] J. M. Zhang and D. Y. Shen, "A Novel Substrate Used for Simultaneous SERS and RAIR," Chinese Chemical Letters, Vol. 13, No. 6, 2002, pp. 563-566.

[15] J. B. Jackson and N. J. Halas, "Surface-Enhanced Raman Scattering on Tunable Plasmonic Nanoparticles Substrates," Proceedings of the National Academy of Sciences of the United States of America, Vol. 101, No. 52, 2004, pp. 17930-17935. doi:10.1073/pnas.0408319102

[16] E. Horváth, Gy. Kátay, E. Tyihák, J. Kristóf and A. Rédey, "Critical Evaluation of Experimental Conditions Influencing the Surface-Enhanced Raman Spectroscopic (SERS) Detection of Substances Separated by Layer Liquid Chromatographic Techniques," Chromatographia, Vol. 51, No. 1, 2000, pp. S297-S301. doi:10.1007/BF02492821

[17] R. Y. Sato-Berrú, J. Medina-Valtierra, C. Medina-Gutierrez and C. Fraustro-Reyes, "Quantitative NIR-Raman Analysis of Methyl-Parathion Pesticide Microdroplets on Aluminum Substrates," Spectrochimica Acta Part A, Vol. 60, No. 10, 2004, pp. 2231-2234. doi:10.1016/j.saa.2003.11.021

[18] A. E. Siegman, "Lasers," University Science Books, Sausalito, 1986.

[19] G. Placzek, "Rayleigh-Streuung und Raman- Effekt in Handbuch der Radiologie," In: E. Marx, Ed., Translation: The Rayleigh and Raman Scattering (University of California Radiation Laboratory (UCRL), Trans 526(L), 1962), Acadeische-Verlag, Leipzig, 1934, p. 205.

[20] D. A. Long, "Raman Spectroscopy" McGraw-Hill, New York, 1977.

[21] A. G. Ryder, G. M. O’Connor and T. J. Glynn, “Quantitative Analysis of Cocaine in Solid Mixtures Using Raman Spectroscopy and Chemometric Methods," Journal of Raman Spectroscopy, Vol. 31, No. 3, 2000, pp. 221-227. doi:10.1002/(SICI) 1097-4555(200003)31:3<221::AID-JR S518>3.0.CO;2-5

[22] D. Pratiwi, J. P. Fawcett, K. C. Gordon and T. Rades, "Quantitative Analysis of Polymorphic Mixtures of Ranitidine Hydrochloride by Raman Spectroscopy and Principal Components Analysis," European Journal of Pharmaceutics and Biopharmaceutics, Vol. 54, No. 3, 2002, pp. 337-341. doi:10.1016/S0939-6411(02)00113-3 